\title{
Concluding summary
}

* Very often, in collision problems two kinds of behaviour are considered as opposite: static and dynamical. In a static process, a few particles are brought together during the collision time and the interaction does not depend on collision velocities. In a dynamical process, the transitions are induced by the relative motion of the particles during the collision time, and the interactions are then increasing with the collision velocity. For bound state continuum transitions, the traditional picture was a static one: when brought together the collision partners form a quasistationary state that decays into the continuum, in an irreversible manner. For electron-molecule collisions, the resonant picture is a static one (the incident electron is captured by the target to form a negative molecular ion that decays by dissociation or electron reemission), while the sudden picture is a dynamical one (the electron bouncing off the molecule kicks the nuclei apart). The above separation between static and dynamical processes is in fact very close to the problem of diabatic or adiabatic representations for atomatom collisions and as a matter of fact a transformation from a diabatic representation to an adiabatic one transforms static processes into dynamical processes. 
The representation of a quasistationary state interacting with a continuum is of the diabatic type; if the corresponding interaction becomes too large, then the diabatic states loose their meaning as intermediates in the collision, and one would rather use an adiabatic representation with only bound and continuum states, in which the bound-free transitions will be of the dynamical type. Indeed, it is always possible to use a diabatic basis for numerical calculations; however, one should not expect the final results to exhibit any diabatic characters. In the case of one electron processes in negative ions this aspect is crucial due to the large interactions.

For s-wave problems, we have seen that a disappearing bound state does not transform into a resonant state, and thus even the static picture consists of continuum states. The bound state-continuum transitions are then induced by the collision velocity; these transitions are not irreversible and an ejected electron can be recaptured later on in the collision.

For p-wave problems, a disappearing bound state transforms into a resonance thus yielding the basis for a static picture. However, the $\mathrm{H}^{-}-\mathrm{H}$ problem we examined in some detail seems to be more on the dynamical side than on the static one.

For higher 1-wave problem, one can expect the resonance aspects (and therefore the static representation) to dominate the processes, because of the decrease of the resonance width. 
This can be observed for example in the $e^{-}-\mathrm{O}_{2}$ vibrational excitation cross sections shown below (Linder et al., 1971, Z.Naturf.26A, 1617). They present a rich structure: each peak is attributed to a vibrational state of the intermediate $\mathrm{O}_{2}{ }^{-}$-ion. The resonance width is small enough to allow for the $\mathrm{O}_{2}{ }^{-}$ molecule performing a few complete vibrations before the electron is ejected.

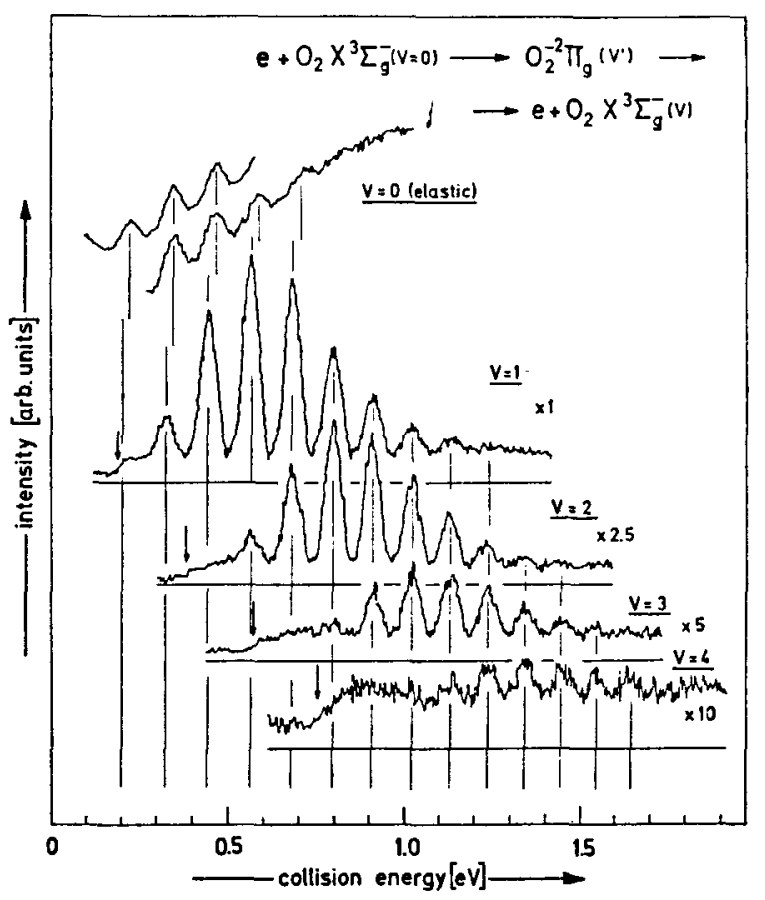


* Model calculations have been performed by Mündel and Domcke (1984,J.Phys.B 17, 3593) for vibrational excitation and dissociative attachment in the $d$ wave case. It can be considered as an intermediate case between the extremely broad resonance like $\mathrm{H}_{2}{ }^{-}$ and the narrow resonance allowing the observation of discrete levels like $\mathrm{O}_{2}{ }^{-}$. The following figure shows the potential energy curves of their model for the $\mathrm{AB}$ (long broken curve) and $\mathrm{AB}^{-}$ systems. The short broken curve is the discrete level potential energy, named $E_{d}(R)$ in the formalism presented in Chapter 5 , and the full line is the resonance energy.

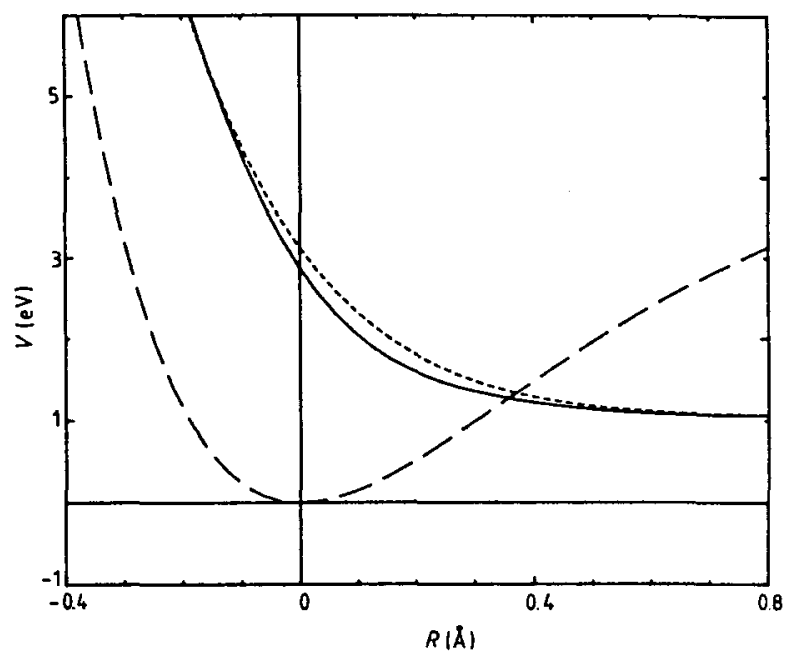


The next figure presents their results for the elastic scattering $(a: 0 \rightarrow 0)$, vibrational excitation $(b: 0 \rightarrow 1$ and $c: 0 \rightarrow 2)$ and dissociative attachment (d) cross sections. It presents both the exact non local results (full curve)and the predictions of the local complex potential approximation (full curve with circles). The quality of the LCP results is much better than that encountered in the broad resonance case $\left(e^{-}-H_{2}\right)$; in particular the DA process is quite well represented.

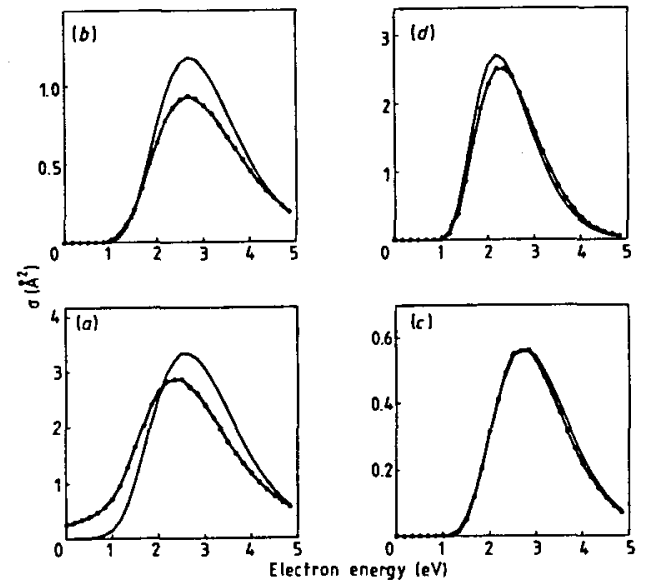

The failure of the LCP in the $0 \rightarrow 0$ channel at low energy is due to the incorrect threshold behaviour of the local approximation (see chapter 2: at low energy the width has to depend on the energy). Domcke and Mündel also present a second model calculation where the negative ion energy crosses the neutral energy around the equilibrium position; it is generated from the previous model by simply shifting downwards the negative ion energy curve: 


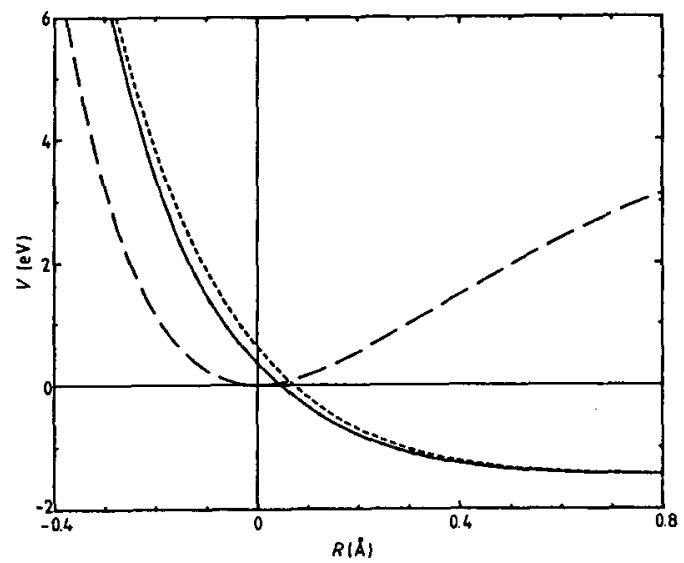

Their results (exact non local: full line, and local complex potential approximation: full line with circles) are presented beLow $(a, b$ and $c$ are the $0 \rightarrow 0,0 \rightarrow 1$ and $0 \rightarrow 2$ vibrational excitation cross sections and d shows the dissociative attachment cross sections). The LCP results are now much worse than in the present example; in particular, the LCP dissociative attachment cross section is diverging at zero energy instead of vanishing (see discussion of DA threshold laws in chapter 6).
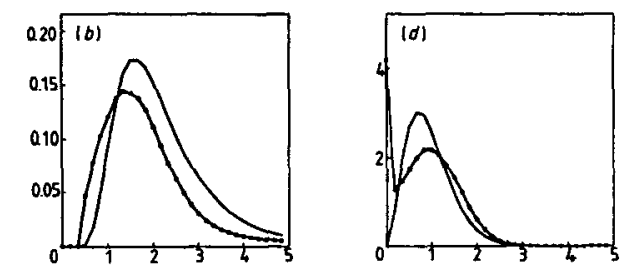

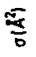
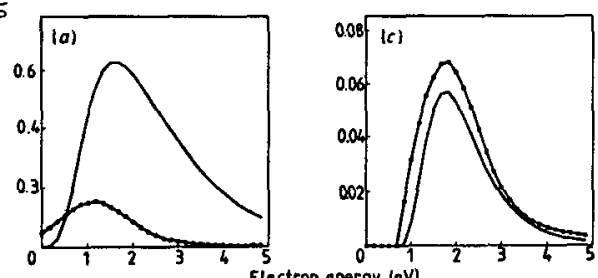

Eiectron energy (eV) 
This has to be attributed to the very low position of the resonance, close to threshold at the equilibrium position. For low energy collisions, the width of the resonance has to be energy dependent (see chapter 2): indeed a resonant scattering amplitude is of the form $\left(E_{r}\right.$ and $\Gamma$ are the position and width of the resonance):

$$
\mathrm{f}=\frac{1}{\mathrm{k}} \frac{\Gamma / 2}{\mathrm{E}_{\mathrm{x}}-\mathrm{E}-\frac{\mathrm{i} \Gamma}{2}}
$$

When the collision energy $E$ goes to zero, the scattering amplitude does not vanish as it should. The threshold analysis of such an amplitude presented in chapter 2 showed that the width $\Gamma$ should be energy dependent $\left(\Gamma \propto \mathrm{E}^{\mathrm{l}+1 / 2}\right)$, so that the resonant amplitude exhibits the correct behaviour as $E \rightarrow O\left(f \propto E^{l}\right)$. The energy dependence of the width is not taken into account in a local description, which then fails close to a threshold. So, even though a resonant picture might seem appropriate for such a problem with a moderate resonance width, the applicability of the local complex potential approximation should be restricted to energy regions not too close to a threshold. An improvement of the LCP has been proposed by Bardsley ("semilocal" approximation in Bardsiey 1979, Electron molecule and photon molecule collisions ed. T.Rescigno, V.MC Koy and B.Schneider, Plenum Press p.267-80). Here, the resonance width and shift are treated as local quantities while keeping the correct energy dependence of the ingoing and outgoing amplitudes. The method yields cross sections exhibiting the correct threshold behaviour. 
* The effective range approximation has been studied in quite some details. It applies to the situation of a low energy electron interacting with a molecule. The basic idea of the method is to use a very simple one-electron model which represents the real many-electron system in the limit of low energies. The ERT representation of electron molecule interactions proved itself to be simple enough to allow for an exact treatment of boundstate continuum transitions in the course of the collision. Thisi approach is favourable in the ase of complex collision problems, i.e., instead of aiming at a detailed and sophisticated description of the collision system at the expense of having to leave out certain important aspects in the collision treatment, one should look for a simplified description of the collision system which makes the treatment of the collision problem manageable. 